\title{
Effect of acute heat exposure on skin blood flow of the paralyzed thigh in persons with spinal cord injury
}

\author{
M Yamasaki*,1, M Shiokawa ${ }^{2}$, SW Choi $^{3}$ and S Muraki ${ }^{4}$ \\ ${ }^{1}$ Department of Health Science, Hiroshima University, Hiroshima, Japan; ${ }^{2}$ Department of Physical Therapy, \\ Hiroshima Prefectural College of Health Sciences, Mihara, Japan; ${ }^{3}$ Department of Physical Education, Duksung \\ Women's University, Seoul, Korea; ${ }^{4}$ Department of Nutrition and Health Science, Siebold University of Nagasaki, \\ Nagasaki, Japan
}

\begin{abstract}
Objectives: To investigate whether increased body temperature during heat exposure promotes skin blood flow of the thigh (SBFT) in individuals with spinal cord injury at rest. Study design: A prospective study with high lesion and with low lesion.

Setting: Hiroshima University, Higashi-Hiroshima, Japan.

Methods: Seven male paraplegics (T5-L2) and seven able-bodied subjects volunteered to participate in the experiment. First the subject rested for $1 \mathrm{~h}$ at an ambient temperature (Ta) of $25^{\circ} \mathrm{C}$ and a relative humidity $(\mathrm{Rh})$ of $50 \%$. Thereafter, the subject moved to a climatic chamber heated to $\mathrm{Ta}$ of $33^{\circ} \mathrm{C}$ an $\mathrm{Rh}$ of $50-55 \%$ and was exposed to this environment for $1 \mathrm{~h}$. SBFT, stroke volume, heart rate, the skin temperature of the medial thigh (Tthigh) and the tympanic membrane temperature (Tty) were assessed during the experiment.

Results: The four subjects with high lesions between Th6 and Th10 (HL) showed no increase in SBFT, while the remaining three subjects with low lesion at Th11 or Th12 (LL) showed increased SBFT in the hot environment. Although Tty increased with exposure time in heat, correlation between Tty and SBFT was not significant in either paraplegic group. However, correlation between SBFT and Tthigh was highly significant in both groups. The increase in Tthigh in LL was due to increased skin blood flow in the thigh, while in HL it was considered attributable to heat transfer from the environment to the skin.

Conclusion: These findings indicate that SBFT during heat exposure was largely dependent on the level of spinal cord injury. SBFT with low lesions increased during heat exposure when the sympathetic cutaneous vasomotor supply of the thigh was preserved.
\end{abstract}

Spinal Cord (2000) 38, 224-228

Keywords: paraplegics; heat exposure; skin blood flow; tympanic membrane temperature; skin temperature

\section{Introduction}

It has been suggested that the circulation below the spinal cord injury in paraplegics and quadriplegics plays an important role in circulatory regulation during exercise. ${ }^{1-6}$ For this reason, some recent studies have focused on skin blood flow (SBF) in the paralyzed lower limbs to investigate blood circulation of individuals with spinal cord injury. ${ }^{7-9}$ In general, blood circulation in the skin of the lower limbs in spinal cord injury (SCI) can be promoted by submaximal exercise ${ }^{7}$ or heat stress $^{10}$ if the injury level of spinal cord is below L1. In addition, it was also demonstrated that SBF of the thigh (SBFT) in low lesions (T12 to L5) began to elevate markedly during submaximal exercise when the tympanic membrane temperature (Tty) exceeded a threshold temperature of $36.69^{\circ} \mathrm{C}^{8}$

*Correspondence: M Yamasaki, Department of Health Science; Hiroshima University, 1-7-1 Kagamiyama, Higashi-Hiroshima 7398521, Japan
However, previous investigations have clearly shown that the higher body temperature of SCI subjects in a hot environment is due to the lack of peripheral circulation control in comparison to that in healthy subjects. ${ }^{11-14}$ Attia and Engel ${ }^{11}$ suggested that the body temperature setpoint was variable and a function of ambient conditions. From these investigations, therefore, individuals with SCI can be assumed to be partially poikilothermic. Accordingly it is naturally deemed that the Tty instantly increases over the threshold of $36.69^{\circ} \mathrm{C}$ in a hot environment even at rest. If the increase in core temperature, eg Tty, is essential for SBFT in those with low lesions, their SBFT will increase when exposed in a hot environment. Furthermore, it is also established that during heat exposure, skin temperature increased regardless of the level of spinal cord injury. ${ }^{11,13,15}$ If the increased skin temperature of those exposed to heat directly stimulates cutaneous blood vessels, ${ }^{16}$ this mechanism is 
considered to affect SBFT in high lesions where sympathetic outflows from spinal cord are completely isolated from the brain. However, there are no studies where the relationships between core and skin temperature and the SBFT have been investigated during heat exposure.

The present study, therefore, examined whether increased body temperature during heat exposure promotes SBFT at rest. Because it is necessary to discuss the circulatory and thermoregulatory responses to clarify the effect of heat exposure on SBFT in SCI, Tty, the skin temperature of the thigh (Tthigh) and cardiac output (Q) were monitored during the experiment.

\section{Methods}

\section{Subjects}

Seven male paraplegic subjects volunteered to participate in the present experiment. The general characteristics of these subjects are shown in Table 1. All were disabled by a complete lesion between T5 and L 2 but were otherwise healthy. In addition, seven male able-bodied subjects (ABS) served as controls. Controls ranged in age from $24-48$ years, in body mass from $64-78 \mathrm{~kg}$, and in height $165-185 \mathrm{~cm}$. All participants gave written informed consent before participating in the study.

\section{Testing protocols}

Experiments were conducted during July, August and September when the subjects should have been naturally heat acclimated. Each subject was tested at the same time of day between 1300 and $1600 \mathrm{~h}$ to minimize the possibility of any time effects. All subjects were asked to refrain from eating and drinking during the $2 \mathrm{~h}$ preceding the experiments.

The subject reported to the laboratory dressed in shorts, singlet, shoes and socks. First the subject was weighed and several electrodes were attached to measure SBFT, heart rate (HR), stroke volume (SV) and Tthigh. Then, the subject rested in his wheelchair or a chair for $1 \mathrm{~h}$ at an ambient temperature (Ta) of $25^{\circ} \mathrm{C}$ and a relative humidity ( $\left.\mathrm{Rh}\right)$ of $50 \%$. Immediately thereafter, the subject moved to a

Table 1 Characteristics of paraplegics

\begin{tabular}{lccccc}
\hline Subject & $\begin{array}{c}\text { Age } \\
(\text { years })\end{array}$ & $\begin{array}{c}\text { Body mass } \\
(\mathrm{kg})\end{array}$ & $\begin{array}{c}\text { Height } \\
(\mathrm{cm})\end{array}$ & $\begin{array}{c}\text { Injury } \\
\text { level }\end{array}$ & $\begin{array}{c}\text { Years since } \\
\text { injury }\end{array}$ \\
\hline A & 34 & 54.0 & 163 & Th6 & 15 \\
B & 46 & 83.8 & 170 & Th6 & 18 \\
C & 47 & 45.3 & 160 & Th7, 8 & 22 \\
D & 26 & 60.6 & 182 & Th9, 10 & 2 \\
E & 43 & 88.7 & 183 & Th11 & 6 \\
F & 44 & 78.8 & 176 & Th12 & 20 \\
G & 50 & 57.1 & 162 & Th12 & 24
\end{tabular}

climatic chamber that was already heated to $\mathrm{Ta}$ of $33^{\circ} \mathrm{C}$ and $\mathrm{Rh}$ of $50-55 \%$. The subject was exposed to the heat environment for $1 \mathrm{~h}$ sitting in his wheelchair or a chair.

\section{Physiological variables}

All physiological measurements were monitored starting after $30 \mathrm{~min}$ at $25^{\circ} \mathrm{C}$ and continuing until the end of the experiment with a 5-min interruption to move to the hot environment.

SBFT was continuously monitored with a laserDoppler flowmeter (ALF 21, Advance Co., Tokyo, Japan). The laser-Doppler probe was attached at the center of the anterior thigh. The laser signals were recorded by a digital printer (ALF-P1, Advance Co., Tokyo, Japan). The time interval of measurement was set at $1 \mathrm{~min}$.

SV and HR were measured by an impedance cardiography (Impedance Plethysmograph 4143, NEC-Sanei, Tokyo, Japan) connected with MacLab/ 8e (AD Instruments Pty Ltd., Castle Hill, Australia) to obtain a differentiated impedance cardiogram. All data of the impedance cardiogram were consecutively recorded on a personal computer. The technique employed was essentially the same as that described by Miles and Gotshall. ${ }^{17}$ Ten continuous cardiac cycles every $10-\mathrm{min}$ were used to calculate mean SV. $\mathrm{Q}$ was obtained by $\mathrm{SV} * \mathrm{HR}$.

Tthigh was measured once every minute by a thermocouple at the site of the medial thigh near the measuring position of SBFT. The tympanic membrane temperature was recorded once every 5 -min by an infrared thermometer (FirstTemp Genius 3000A, Sherwood Medical, St. Louis, USA).

\section{Statistical analysis}

The SCI subjects were classified into two groups by SBFT response. The means of physiological measurements were calculated for each group and ABS. The mean values were then utilized for data analysis. To clarify the relation between Tty and Tthigh and SBFT, regression analysis was used for these data. A probability level of 0.05 or less was considered significant.

\section{Results}

Figure 1 shows the ratio of SBFT to baseline SBFT measurement at $25^{\circ} \mathrm{C}$ in SCI and ABS during the course of the experiment. The SBFT of ABS strikingly and continuously increased during heat exposure. However, the response patterns in SCI were apparently dependent on the level of injury. The four subjects (subjects A, B, C and D) showed no increase in SBFT during the heat exposure. The injury levels of these subjects were relatively high between Th6 and Th10. The remaining three subjects (subjects E, F and G) with low lesion at Th11 and Th12 showed an 
increase in SBFT in heat, although the increase was not as remarkable as that in ABS. Thus, the subjects were divided into two groups according to the pattern of SBFT response; HL (subjects $\mathrm{A}-\mathrm{D}$ with high lesion) and LL (subjects E-G with low lesion).

Figure 2 represents the mean Tty in each of three groups. At $25^{\circ} \mathrm{C}$ ABS showed somewhat higher Tty in comparison with that of the paraplegic groups. During exposure to $33^{\circ} \mathrm{C}$, the $\mathrm{Tty}$ of each group gradually increased and at the end of the exposure every group showed a similar Tty. Regression analyses were performed in which Tty was related to SBFT (Figure 3). As Tty was increased above $37.5^{\circ} \mathrm{C}$ in $\mathrm{ABS}$, the relationship became definitely curvilinear in nature with the curves rounding off at the higher values. The total relationship, therefore, took the form of ' $\mathrm{j}$ ' shaped curve in ABS. However, the linearity of the regression line was rejected in both paraplegic groups.

Figure 4 represents the mean Tthigh in each of the three groups. Although the Tthigh of ABS during exposure to $33^{\circ} \mathrm{C}$ was a little lower than those of other groups, the remarkable difference was not found in the mean Tthigh. When SBFT was plotted in relation to

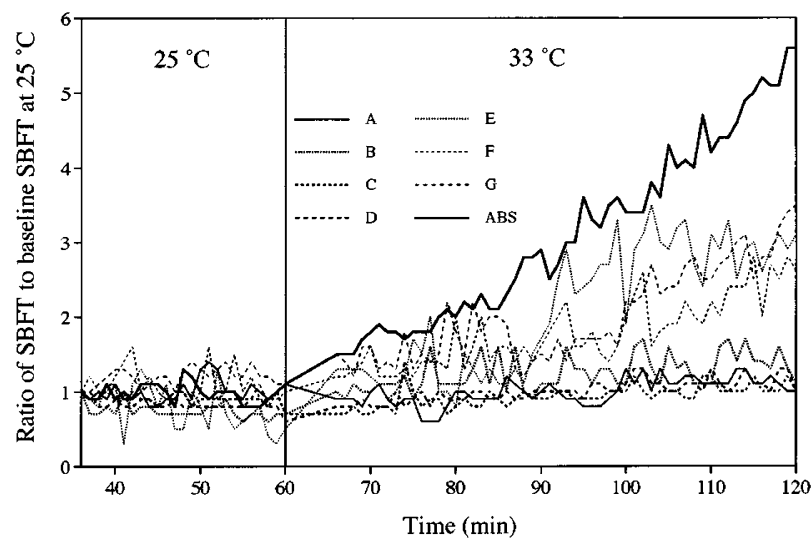

Figure 1 Time course of the ratio of SBFT to baseline SBFT measurement in each paraplegic and ABS

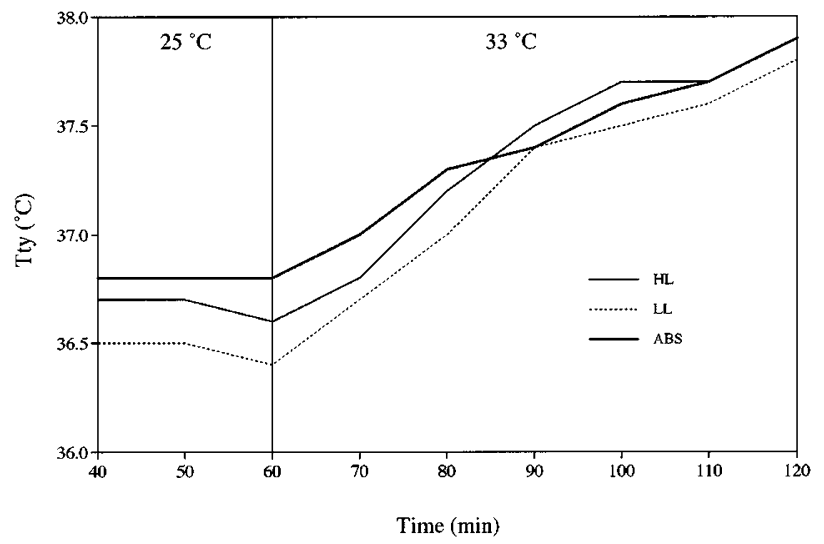

Figure 2 Time course of mean Tty in the three groups
Tthigh during heat exposure, the regression lines obtained differed from each other in all three groups (Figure 5). In Figure 5, all mean values of Tthigh and SBFT obtained every $1 \mathrm{~min}$ in each group during heat exposure were used. A relatively rectilinear relationship was found between Tthigh and SBFT in HL. In contrast, LL and ABS showed a curvilinear rather than linear relationship. The results for ABS presented slowly rising curves up to Tthigh of $34^{\circ} \mathrm{C}$, then the curves began rising sharply. Correlations between SBFT and Tthigh were highly significant in all groups.

$\mathrm{HR}$ and $\mathrm{SV}$ responses in HL were very similar to those in LL. Average HR in HL and LL were 67.3 and 66.2 at $25^{\circ} \mathrm{C}$ and 73.0 and 72.1 beats. $\min ^{-1}$ at the end of heat exposure, respectively. SV of $\mathrm{HL}$ and $\mathrm{LL}$ showed a relatively constant value of about $70 \mathrm{ml} \cdot$ beat $^{-1}$ during the experiment. In ABS, both $\mathrm{HR}$ and SV were greater than those in HL and LL. Average $\mathrm{HR}$ of ABS was 71.7 at $25^{\circ} \mathrm{C}$ and 77.6

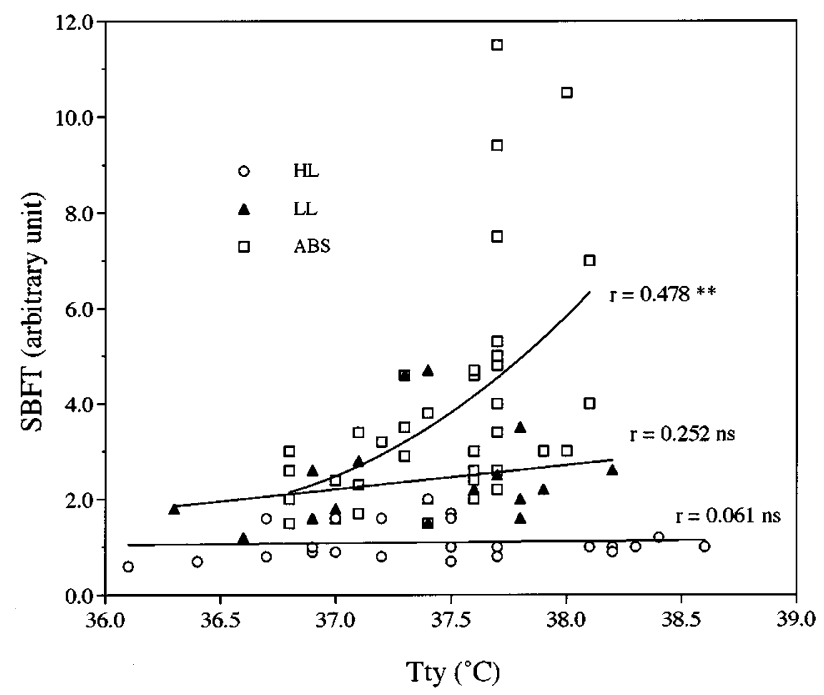

Figure 3 The relationship between Tty and SBFT during heat exposure in the three groups

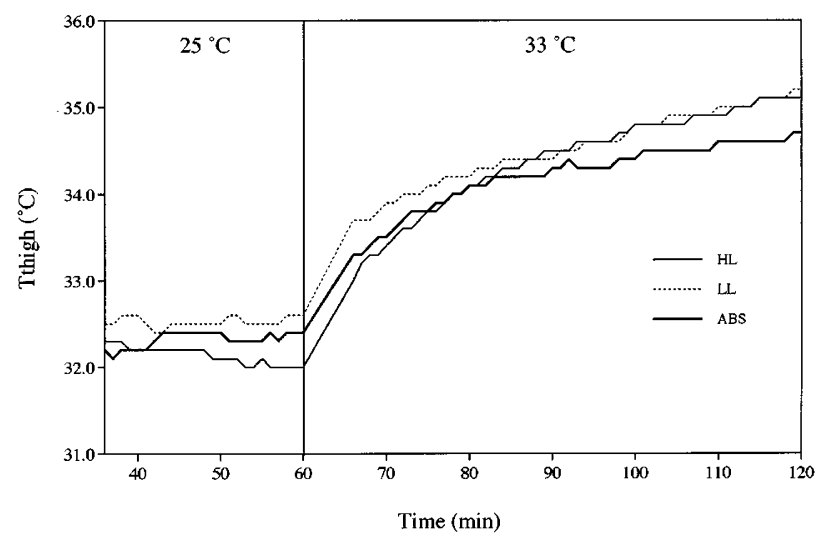

Figure 4 Time course of mean Tthigh in the three groups 


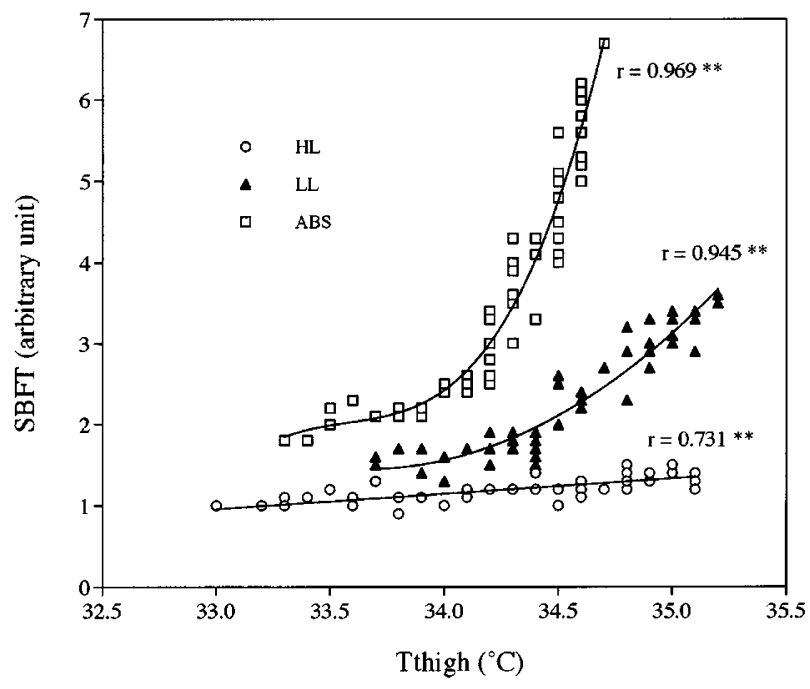

Figure 5 The relationship between Tthigh and SBFT during heat exposure in the three groups

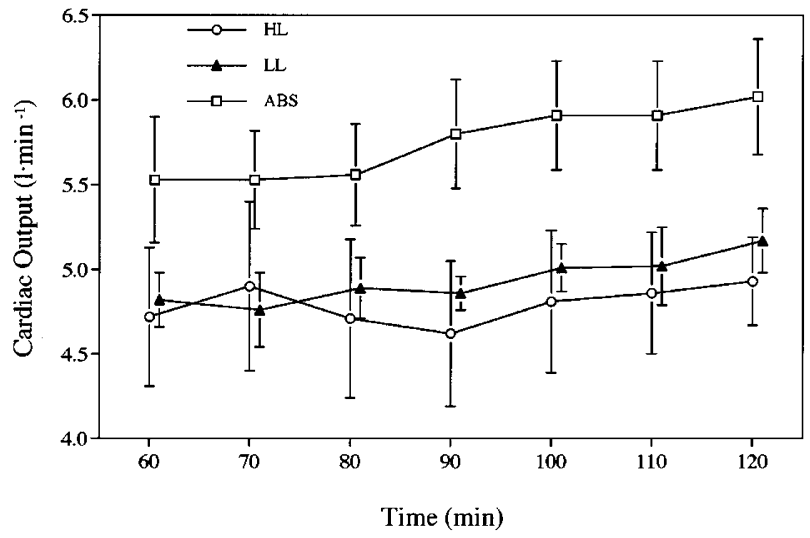

Figure 6 Time course of mean $\mathrm{Q}$ in the three groups. Vertical bar indicates the standard error of mean

beats. $\min ^{-1}$ at the end of heat exposure, while SV ranged between 77.0 and $79.5 \mathrm{ml} \cdot$ beat $^{-1}$. Figure 6 shows mean $\mathrm{Q}$ in each group during heat exposure. ABS developed $Q$ ranging from $5.6-6.21 \cdot \mathrm{min}^{-1}$ during the experiment. On the other hand, there were no noticeable increases in $\mathrm{Q}$ during heat exposure in HL and LL.

\section{Discussion}

In ABS who showed a slight increase in Q, SBFT increased abruptly with the exposure time. However, the present study clearly showed that the SBFT in SCI subjects during heat exposure is dependent on the level of spinal cord injury. Muraki et $a l^{8}$ demonstrated increased SBFT with lesions at L1 or below irrespective of exercise intensity, while there was no increase with lesions at or above Th12 during a brief arm cranking exercise $(6 \mathrm{~min})$. These findings were not completely consistent with our present results that the SBFT in SCI with lesions between Th6 and Th10 remained unchanged and that in SCI with low lesions at Th11 and Th12 increased with exposure time, although the SBFT in SCI with a low lesion was much lower than that in ABS. However, when exercise time was prolonged for $30 \mathrm{~min}$ at a relatively light work load of 20 watts, the SBFT of two SCI subjects with a lesion at Th12 increased significantly during exercise. ${ }^{7}$ In that study, ${ }^{7}$ SCI with lesions between Th7 and Th11 were not tested. Therefore, it was not clear if these results were in good agreement with the present findings, but as Normell ${ }^{10}$ pointed out, in contrast with high lesions, low lesions showed a cutaneous vasomotor response in the anterior thigh. The border of this response was considered to be around Th10, because the response was preserved in some lesions at Th10 and Th11 and absent with lesions at Th8. This was consistent with our results that SCI with lesions below Th11 showed an increase in SBFT during heat exposure.

Highly significant correlations were found between SBFT and Tthigh in all groups in contrast to those between SBFT and Tty. SBF in augmented as a function of the internal body temperature and skin temperature in healthy humans. ${ }^{18}$ It has been shown that SBFT in low lesions begins to elevate markedly and was significantly related to Tty when the Tty exceeded a threshold temperature of $36.69^{\circ} \mathrm{C}$ during arm cranking exercise at an ambient temperature of $25^{\circ} \mathrm{C},{ }^{7}$ although there were no significant relationships between SBFT and Tty in either HL or LL in this study. At an ambient temperature of $25^{\circ} \mathrm{C}$ Tthigh remains relatively low even during exercise. As seen in Figure 4 the Tthigh immediately increased during heat exposure at $33^{\circ} \mathrm{C}$ in all groups. Possibly Tthigh has a greater effect on SBFT than Tty during heat exposure.

The SBFT of HL remained unchanged during heat exposure regardless of increase in either Tthigh or Tty, because HL have impaired sympathetic vasomotor function in the lower limb. ${ }^{8-10,14,19}$ However, Tthigh of HL increased constantly with the exposure time, as did those of LL and ABS. Attia and Engel ${ }^{11}$ indicated that rectal and skin temperatures in subjects with SCI showed greater variation than those in ABS with variations in ambient temperature. From those findings, they concluded that SCI subjects exposed to heat might possess a thermoregulatory setpoint which varies directly with ambient thermal conditions; that is, a state of partial poikilothermia. This phenomenon must be more prominent with high lesions than those with low lesions, because body temperature regulation of the former was inferior to the latter. In a state of partial poikilothermia, SCI subjects received heat from the environment and Tthigh increased without increment in SBF.

In conclusion, circulatory responses in individuals with SCI during heat exposure were dependent on the level of spinal cord injury. In LL, SBFT increased, 
while that in HL remained unchanged in a hot environment despite increased Tthigh. In those with low lesions whose sympathetic cutaneous vasomotor at the thigh was preserved, SBFT increased during heat exposure.

\section{References}

1 Davis GM. Exercise capacity of individuals with paraplegia. Med Sci Sports Exerc 1993; 25: 423 - 432.

2 Figoni SF. Exercise responses and quadriplegia. Med Sci Sports Exerc 1993; 25: $433-441$.

3 Hopman MTE, Oeseburg B, Binkhorst RA. Cardiovascular responses in paraplegic subjects during arm exercise. Eur $J$ Appl Physiol 1992; 65: $73-78$.

4 Hopman MTE, Verheijen PHE, Binkhorst RA. Volume changes in the legs of paraplegic subjects during arm exercise. $J$ Appl Physiol 1993; 75: 2079-2086.

5 Hopman MTE. Circulatory responses during arm exercise in individuals with paraplegia. Int J Sports Med 1994; 15: 126 - 131 .

6 Kinzer SM, Convertino VA. Role of leg vasculature in the cardiovascular response to arm work in wheelchair-dependent populations. Clin Physiol 1989; 9: 525-533.

7 Muraki S et al. Relationship between temperature and skin blood flux in lower limbs during prolonged arm exercise in persons with spinal cord injury. Eur J Appl Physiol 1996; 72: 330-334.

8 Muraki S et al. Effect of arm cranking exercise on skin blood flow of lower limb in people with injuries to the spinal cord. Eur J Appl Physiol 1995; 71: 28-32.
9 Muraki S et al. Effect of maximal arm exercise on skin blood flux in the paralyzed lower limbs in persons with spinal cord injury. Eur J Appl Physiol 1996; 74: 481-483.

10 Normell LA. Distribution of impaired cutaneous vasomotor and sudomotor function in paraplegic man. Scand J Clin Lab Invest 1974; 33: $25-41$

11 Attia M, Engel P. Thermoregulatory set point in patients with spinal cord injuries (spinal man). Paraplegia 1983; 21: 233-248.

12 Downey JA, Huckaba CE, Myers SJ, Darling RC. Thermoregulation in the spinal man. $J$ Appl Physiol 1973; 34: 790-794.

13 Downey JA et al. Sweating responses to central and peripheral heating in spinal man. J Appl Physiol 1976; 40: $701-706$.

14 Guttmann L, Silver J, Wyndham CH. Thermoregulation in spinal man. J Physiol 1958; 142: 406-419.

15 Huckaba CE et al. Sweating responses of normal, paraplegic and anhidrotic subjects. Arch Phys Med Rehabil 1976; 57: 268-274.

16 Randall WC, Wurster RD, Lewin RJ. Responses of patients with high spinal transection to high ambient temperatures. $J$ Appl Physiol 1966; 21: $985-993$.

17 Miles DS, Gotshall RW. Impedance cardiography: noninvasive assessment of human central hemodynamics at rest and during exercise. Exerc Sport Sci Rev 1989; 17: 231-263.

18 Wenger CB, Roberts MF, Stolwijk JAJ, Nadel ER. Forearm blood flow during body temperature transients produced by leg exercise. J Appl Physiol 1975; 38: 58-63.

19 Cooper KE, Ferres HM, Guttmann L. Vasomotor responses in the foot to raising body temperature in the paraplegic patient. $J$ Physiol 1957; 136: $547-555$ 